\title{
A Picocellular CDMA/TDD Overlay on GSM
}

\author{
Piotr Kaczorek ${ }^{*}$, Dominik Rutkowski ${ }^{* *}$ \\ "Department of Marine Radio Electronics, Gdynia Maritime Academy, Poland \\ "* Department of Radio Communication Systems, Technical University of Gdansk, Poland \\ pik@wsm.gdynia.pl, nick@pg.gda.pl
}

Key words: $\quad$ GSM system , CDMA/TDD, UMTS/IMT2000

Abstract: $\quad$ To improve the utilisation of frequency bands assigned to GSM system, an idea of CDMA overlay seems to be interesting. In this paper a picocellular CDMA/TDD overlay on GSM system has been considered. It is corresponding to a TDD mode of WCDMA proposed by ETSI, ARIB and TIA for the future third generation UMTS/MMT2000 system. The overlay is proposed for indoor operation in the area of macrocell of GSM system. As it turns out the implementation of overlay can significantly increase the spectrum efficiency in the frequency band that is shared by GSM system and the overlay. The picocellular CDMA/TDD overlay can be used both for speech and data transmission.

\section{INTRODUCTION}

The frequency band assigned to each radio communication system is a valuable resource and as time goes by we will observe more frequently the coexistence of autonomous radio communication systems sharing the same frequency band with the constrained level of mutual interference.

In general, by CDMA overlay we understand a mobile communication system based on DS-CDMA technique that is sharing frequency band with another mobile communication system based on TDMA or FDMA technique. In the literature one can find many publications in this topics [59]. However, only macrocellular overlay, covering fully the area of GSM cells, has been considered so far. The paper [10] analyses the CDMA overlay in an indoor picocell that is located in the area of a GSM macrocell. Such an overlay has the potential of enhancing the overall spectrum 
efficiency and is able to provide additional capacity in the areas of excessively large traffic, e.g. business districts and commercial centres. Clearly, the condition that must be met to use an overlay is the simplicity of implementation. This is why in [10] an overlay based on WCDMA standard, worked out by ETSI for UMTS/IMT-2000 system, has been proposed. Of course, CDMA overlay should be designed in such a way as to avoid excessive interference to GSM system and it should not restrict in any form GSM system's functioning.

Thus, the chance of using the overlay and its capacity depends upon the mutual interference between GSM system and the overlay. The analysis of such interference in case of CDMA/FDD overlay proposed in [10] has shown that important factor limiting a location and capacity of picocellular overlay is the interference experienced in the uplink of CDMA/FDD overlay and coming from GSM system's mobile terminals.

In the following, a problem of designing a picocellular CDMA overlay on GSM system will be considered provided that WCDMA/TDD standard is employed. The TDD mode enables to increase the range of possible locations of a picocell and to make its capacity independent on location.

\section{CDMA OVERLAY}

Let us assume that frequency band of $12 \mathrm{MHz}$ is assigned to one GSM system operator, the cluster of cells is 4 and the base stations use three-sector antennas. For a uniform traffic in the whole service area each sector of a cell has $N_{T}=5$ frequency channels to its disposal.

The frequency channel of CDMA overlay, having the bandwidth of $4.8 \mathrm{MHz}$, consists of 24 consecutive GSM system's frequency channels in the uplink. Thus, it has two channels, on the average, that are assigned to each sector of GSM system as it is shown in Fig. 1.

Known solutions of macrocellular CDMA overlay consist in narrowband notch filters in the transmitters and receivers of overlay. The characteristics of notch filters used in a given sector of overlay must be chosen adequatly to attenuate frequencies utilised in that sector by GSM system [5-7]. This approach can also be used in the picocellular overlay considered, however, only in such a case when a picocell is located somewhere in the middle of a sector of GSM system's macrocell (see Fig. 1.A). If a picocell is located close to the boundary between sectors, additional filters must be used due to powerful interference coming from the overlay and affecting GSM terminals in the neighbour sector (see Fig. 1.B). These additional filters should attenuate frequencies used in the nearest sector of GSM system. Thus, their characteristics should depend upon specific location of overlay. 


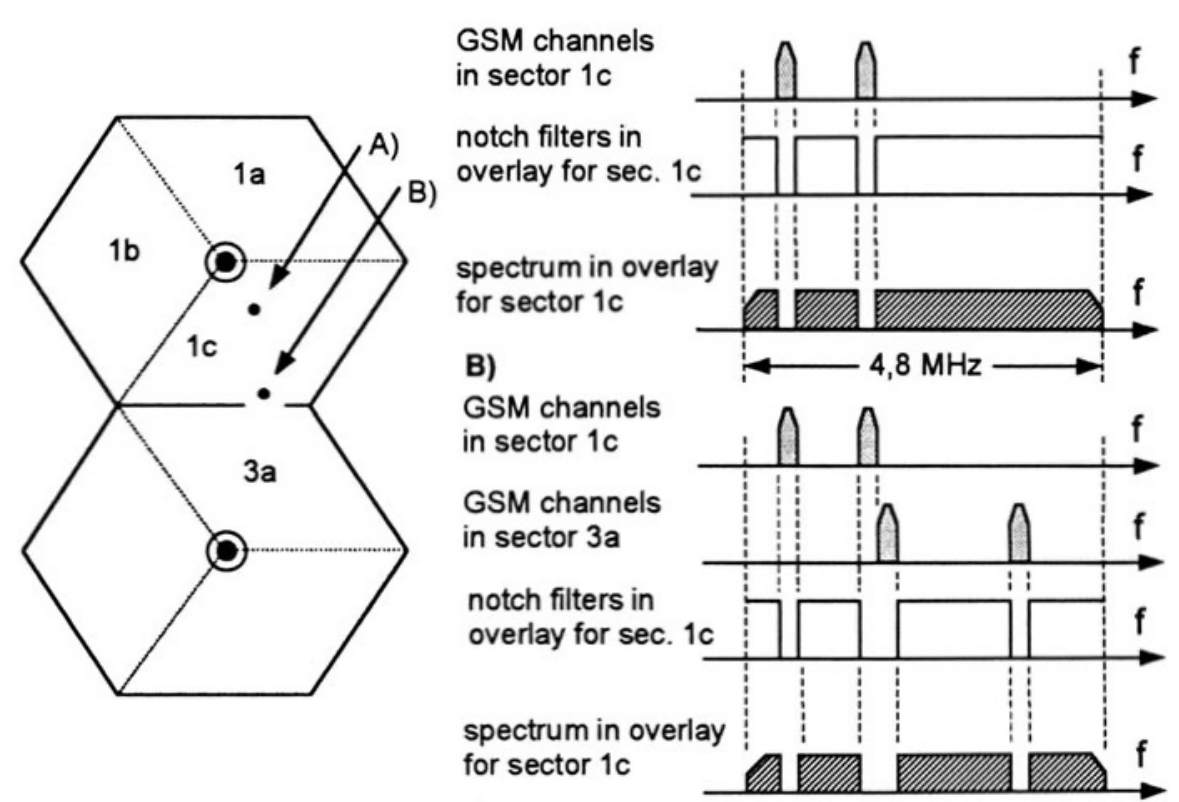

Fig.1. The way of setting up CDMA channel for a picocellular overlay: (A) picocell is located inside a sector of GSM system (B) picocell is located close to sector boundary.

In the following, we are considering the overlay in which filtering is encompassing frequencies used by two GSM sectors, including that with the overlay. It means that filtering will concern at most 4 GSM channels.

\section{TDD MODE IN UMTS/IMT-2000 SYSTEM}

The ETSI proposal of WCDMA standard for UMTS/IMT-2000 system is foreseeing in TDD mode the use of mixed TDMA/CDMA multiaccess scheme. Some of the parameters and characteristics for that mode are given in Table 1.

Table 1. Parameters and characteristics of WCDMA/TDD [1]

\begin{tabular}{|l|c|}
\hline Multiaccess & TDMACDMA \\
\hline TDMA frame & $10 \mathrm{~ms}$ \\
\hline Number of timeslots in TDMA frame & 16 \\
\hline Chip rate & $4,096 \mathrm{Mcps}$ \\
\hline Spreading rate & 16 \\
\hline Channel data rate & $128 \mathrm{ks} / \mathrm{s}(256 \mathrm{~kb} / \mathrm{s})$ \\
\hline Modulation & QPSK \\
\hline
\end{tabular}


As one can see, a $10 \mathrm{~ms}$ TDMA frame is subdivided onto 16 timeslots. Each $625 \mu \mathrm{s}$ timeslot can be assigned for the uplink or downlink. This provides for the flexibility in using TDD mode for different services and environments. Due to CDMA technique more than one burst may be transmitted simultaneously in one timeslot. The orthogonal transmission of different bursts in one timeslot is assured by the use of orthogonal spreading sequences. Of course, different bursts (equivalently spreading sequences) can be assigned to different users or to one user.

For the mode considered two types of bursts have been defined. Further, we are going to analyse TDD mode with type 1 burst which can be transmitted in both uplink and downlink. Its structure is shown in Fig. 2.

\begin{tabular}{|c|c|c|c|}
\hline $\begin{array}{c}\text { Data symbols } \\
61(976 \text { chips) }\end{array}$ & $\begin{array}{c}\text { Midamble } \\
512 \text { chips }\end{array}$ & $\begin{array}{c}\text { Data symbols } \\
61(976 \text { chips })\end{array}$ & $\begin{array}{c}\text { GP } \\
96 \mathrm{CP}\end{array}$ \\
\hline
\end{tabular}

Figure 2. Burst structure of the traffic burst 1 [1]. Notations: GP - guard period with 96 chip periods (CP).

All bursts in all timeslots are spread with the same spreading factor of $G=16$. One can get different transmission rates by assigning different number of timeslots and/or different number of bursts/timeslot for a particular user. Example of mapping $64 \mathrm{kbps}$ data service is given in Fig. 3.

The results of simulation described by ARIB [2] indicate that for indoor $64 \mathrm{kbps}$ LCD (Long Constrained Delay) service the required $\left(E_{b} / N_{o}\right)_{\min }=1.2 \mathrm{~dB}$ and $\left(E_{b} / N_{o}\right)_{\min }=5 \mathrm{~dB}$ for the downlink and uplink, respectively.

One can prove that maximum number of bursts that may be sent in one timeslot is $N_{C B}{ }^{(d n)}=13$ for the downlink and $N_{C B}{ }^{(u p)}=6$ for the uplink, respectively. Through the adequate asymmetric assignment of timeslots between downlink and uplink one can achieve $N_{C}=10$ duplex channels 64 kbps each. 

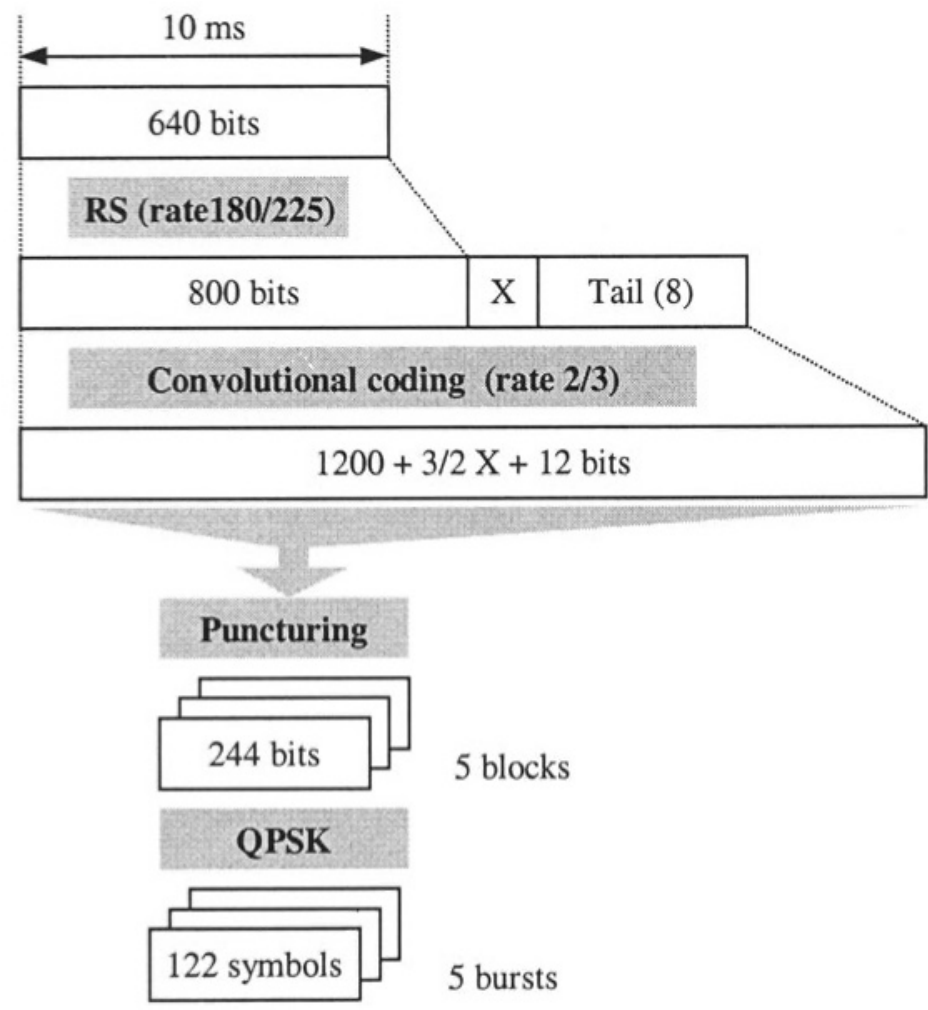

Figure 3. Channel coding for 64 kbps data service [1]. Notations: RS - Reed-Solomon coding, $X$-signalling bits

\section{INTERFERENCE IN CDMA OVERLAY}

The analysis of interference coming from GSM system and affecting CDMA overlay has been carried out for the uplink of CDMA overlay. The way of analysis is similar to the one shown in [10]. The propagation model adopted assumes the path loss proportional to the fourth power of distance. Besides, fading, shadowing, and thermal noise are omitted and ideal power control is employed.

The way of calculation the interference power coming from GSM system is illustrated in Fig. 4. The location of picocell with respect to the base station $\mathrm{BS}_{0}$ of GSM system is described by angle $\varphi_{p}$ and distance $r_{p}$. The source of interference is a base station $\mathrm{BS}_{\mathrm{j}}$ of GSM system that is serving mobile terminal $\mathrm{MS}_{\mathrm{j}}$. The location of $\mathrm{MS}_{\mathrm{j}}$ with respect to $\mathrm{BS}_{\mathrm{j}}$ is given by angle $\gamma_{j}$ and distance $r_{j}$. 


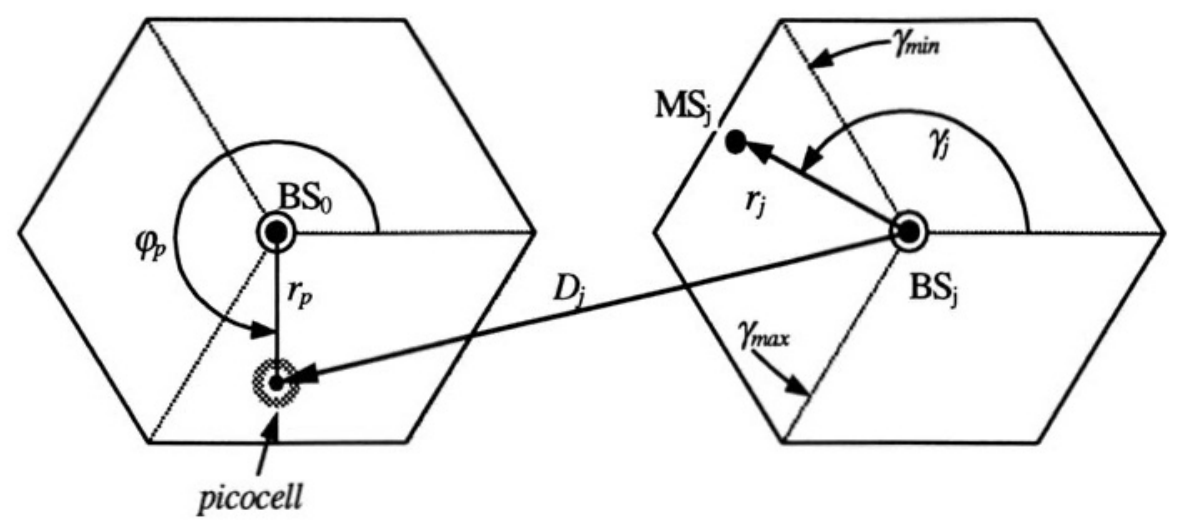

Figure 4. The way of calculation the interference coming from GSM system in the uplink of CDMA overlay.

The average interference power coming from $\mathrm{BS}_{\mathrm{j}}$ that is being received in CDMA overlay depends on the location of picocell. At the assumptions made one can find it as

$$
\overline{I_{j}\left(r_{p}, \varphi_{p}\right)}=\frac{1}{A_{w}} \frac{1}{\frac{\sqrt{3}}{2} R^{2}} \int_{\gamma_{\min }}^{\gamma_{\max }} \int_{0}^{R_{\max }(\gamma)}\left(\frac{r_{j}}{D_{j}}\right)^{\alpha} S_{T} \cdot r_{j} d r_{j} d \gamma_{j}
$$

where $S_{T}$ is the nominal power of signal transmitted by $\mathrm{MS}_{\mathrm{j}}$ and received by $\mathrm{BS}_{\mathrm{j}}, D_{j}$ is a distance between picocell and $\mathrm{MS}_{\mathrm{j}}$. The angles $\gamma_{\min }$ and $\gamma_{\max }$ delimit the sector considered and $R_{\max }(\gamma)$ is a distance between $\mathrm{BS}_{\mathrm{j}}$ and sector boundary. With the assumption made that power control is ideal one, the signal power received by all mobile terminals of GSM system is the same and equal to $S_{T}$. The symbol $A_{w}$ in the expression (1) represents the building penetration loss. In Table 2 typical values of building penetration loss are given. In our considerations more critical value of $A_{w}=10 \mathrm{~dB}$ has been chosen.

Table 2. Building penetration loss [11].

\begin{tabular}{lc}
\hline Environment & Penetration loss (dB) \\
\hline Urban, Suburban & 15 \\
Residential, Rural & 10 \\
\hline
\end{tabular}

One can find the total interference power by summing up the average power for each of the sectors 


$$
I_{T}\left(r_{p}, \varphi_{p}\right)=N_{T / C} \sum_{j} \overline{I_{j}\left(r_{p}, \varphi_{p}\right)}
$$

where $N_{T / C}=2$ results from the conclusion made before that the overlay radio channel of overlay is including on the average two radio channels of each GSM sector (see Fig. 1).

The total interference power is upper-bounded by

$$
I_{T}\left(r_{p}, \varphi_{p}\right) \leq I_{T \max }=0,032 S_{T}
$$

Thus, the carrier-to-interference ratio $C I R$ in the uplink of CDMA overlay is given by

$$
C I R_{C}^{(u p)}=\frac{G S_{C}}{\left(N_{C B}^{(u p)}-1\right) S_{C}+I_{T}}
$$

where $N_{C B}$ is the number of overlay bursts/timeslot, $G$ stands for processing gain and the relationship between nominal power in overlay and GSM system, $S_{C}=0.36 S_{T}$, is calculated similarly to [10].

Since the analysis doesn't take fading, shadowing and thermal noise into account, then we can't directly use $C I R$ for the performance evaluation of overlay. Instead, we can find the degree of degradation of $C I R$ in overlay affected by interference coming from GSM system

$$
\delta C I R_{C}{ }^{(u p)}=\frac{C I R_{C}{ }^{(u p)}{ }_{\left(I_{T}=0\right)}}{C I R_{C}{ }^{(u p)}{ }_{\left(I_{T}\right)}}
$$

Taking (3) and (4) into account, we obtain

$$
\delta C I R_{C}{ }^{(u p)} \leq 0.06 d B
$$

We can draw the conclusion that the influence of GSM system on the overlay is negligible. One can get similar results for the downlink of overlay. We can see that CDMA overlay attains in practice the same capacity as WCDMA system does independently on the location of picocell with respect to the sector of GSM system's macrocell.

\section{INTERFERENCE RANGE OF CDMA OVERLAY}

As we know, in CDMA overlay considered a TDD mode of transmission is used. Thus, the interference coming from overlay affects only downlink of GSM system. In the following, we assume that a picocell with CDMA overlay has a shape of a circle with a radius of $R_{p}$ and the base station is located in the centre. A mobile terminal of GSM system is located at a distance $D_{j}$ from the picocell. This is illustrated in Fig. 5 . 


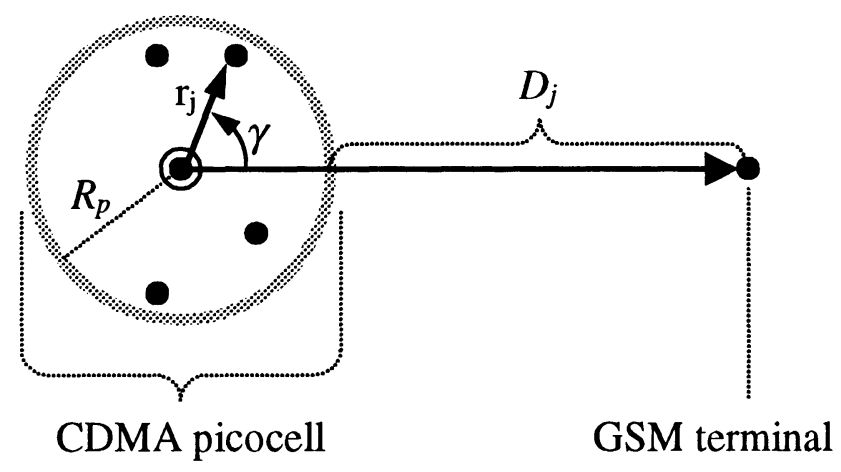

Figure 5. The way of interference range calculation of CDMA overlay.

In view of the assumptions made, the average interference power arriving at the GSM mobile terminal from the base station of overlay can be found from the expression

$$
\overline{I_{C}}=\frac{N_{C B}}{H A_{W}} \frac{1}{\pi R_{p}^{2}} \int_{0}^{2 \pi} \int_{0}^{R_{p}}\left(\frac{r_{j}}{R_{p}+D_{j}}\right)^{4} S_{C} \cdot r_{j} d r_{j} d \gamma
$$

After calculations we get

$$
\overline{I_{C}}=\frac{1}{3} \cdot \frac{N_{C B}}{H A_{W}}\left(\frac{R_{p}}{R_{p}+D_{j}}\right)^{4} S_{C}
$$

where $S_{C}$ is the nominal power of signal received by overlay terminals, $N_{C B}^{(d n)}$ is the number of bursts/spreading sequences transmitted in one timeslot, and $A_{w}$ stands for the building penetration loss.

Due to narrower channel bandwidth in GSM system than in overlay, only a fraction of interference power is affecting GSM terminal. This is reflected in (8) by the interference reduction factor $\mathrm{H}$ given by

$$
H=\frac{W_{C}}{W_{T}}=24
$$

where $W_{C}$ and $W_{T}$ is the channel bandwidth of CDMA overlay and GSM system, respectively.

Now, we can find the carrier-to-interference ratio $C I R_{T}$ in GSM system in the presence of interference coming from CDMA overlay as

$$
C I R_{T}=\frac{S_{T}}{\overline{I_{T}}+\overline{I_{C}}}
$$

The average interference power $I_{T}$ in the downlink of GSM system can be calculated in the same way as in [9]. As a result we obtain 


$$
\overline{I_{T}} \approx 0,0028 S_{T}
$$

Substituting (8) and (11) into (10), we have

$$
C I R_{T}=\frac{S_{T}}{0,0028 S_{T}+\frac{1}{3} \frac{N_{C B}}{H A_{w}}\left(1+\frac{D}{R_{p}}\right)^{-4} S_{C}}
$$

For the same reasons as before we can't use directly formula (12) to the evaluation of interference range for CDMA overlay. Instead, we can find the degree of degradation of $C I R_{T}$ in the GSM mobile terminal caused by interference coming from overlay. As a reference level the value of CIR at the full load of GSM system and lack of interference coming from overlay has been adopted, i.e.

$$
\delta C I R_{T}=\frac{C I R_{T\left(N_{C B}=0\right)}}{C I R_{T\left(N_{C B}, D_{j}\right)}}
$$

The results of calculations are shown in Fig. 6. As one can notice the interference range of CDMA overlay is small and doesn't exceed two radii of picocell for $\delta C l R_{T}=0.1 \mathrm{~dB}$.

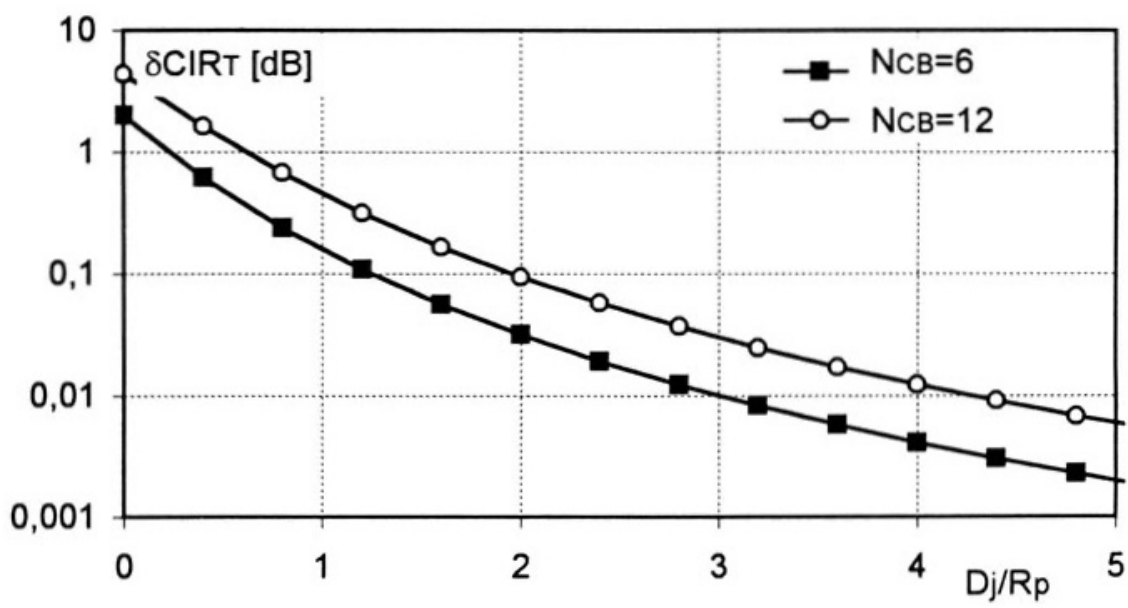

Figure 6. Degradation of CIR vs. $D / R_{p}$ in GSM system due to interference coming from CDMA overlay. Notations: $D_{j}$ - distance between GMS mobile terminal and overlay, $R_{p}-$ radius of picocell, $N_{C B}$ - number of bursts/timesiot.

Having obtained interference range of CDMA overlay one can get the region of permitted picocell locations. In Fig. 7. the boundaries of the regions are shown in which CDMA overlay location is possible in the most unfavourable situation when the radius $R_{p}$ of picocell is 10 times smaller than the radius $R_{m}$ of GSM macrocell. Different boundary lines concern 
different number of filters employed. When filtering in overlay is encompassing only the frequencies of own GSM sector a relatively small area of picocell locations is possible (some $36 \%$ of sector area - see Fig. 7.A).

However, when filtering is including the frequencies of two GSM sectors the allowed area of picocell locations is significantly increased (some $84 \%$ of sector area - see Fig. 7.B). Further increase of the number of filters isn't reasonable since the benefit is small and some degradation of transmission quality in overlay occurs.

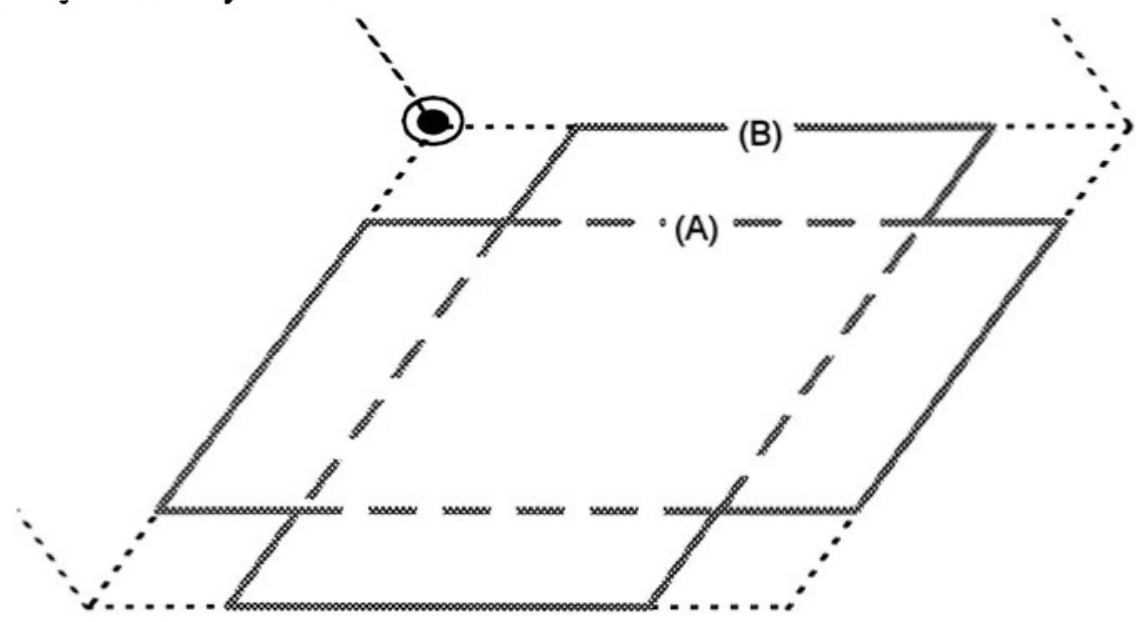

Figure 7. The region of permitted picocell locations.

(A) filtering in overlay of the frequencies of own GSM sector

(B) filtering in overlay of the frequencies of two GSM sectors including the own one.

\section{CONCLUSIONS}

The results obtained suggest that a proposed CDMA overlay can significantly increase the spectrum efficiency of the frequency band shared by GSM system and the CDMA overlay and can provide additional capacity for services.

One can also prove that larger percentage of GSM service area can be used for picocell CDMA overlay with TDD mode than with FDD mode. Apart from this, the implementation of narrowband filters is easier with TDD particularly in the receivers of overlay.

It is also worth to mention that known plans of the evolution and implementation of third generation systems forecast the integration of WCDMA system with GSM system [1-4]. Thus, the proposed overlay can be an attractive platform for the coexistence of GSM and WCDMA system. 
Practical implementation of the proposed CDMA overlay depends on the correct functioning of WCDMA system despite of the filters employed. The results of simulation [5] and hardware tests [8] indicate that this is realisable.

\section{REFERENCES}

[1] ETSI: „UMTS Terrestrial Radio Access (UTRA); Concept evaluation”, Dec. 1997.

[2] ARIB: „Japan's Proposal for Candidate Radio Transmission Technology on IMT-2000: W-CDMA", June 1998

[3] R.Prasad, T.Ojanpera „An Overview of CDMA Evolution Toward Wideband CDMA" IEEE Comm. Surveys, No 4, 1998

[4] J.Schwarz da Silva, B.Barani, B.Arroyo-Fernan-dez „European Mobile Communications on the Move" IEEE Comm. Vol.10, 655-668, Feb.1997

[5] D.M.Gricco, D.L.Shilling „The Capacity of Broadband CDMA Overlaying a GSM Cellular System" Proc. IEEE VTC, 31-35, Stockholm, June 1994

[6] D.L.Schilling et al. „Broadband CDMA overlay” International Journal of Wireless Information Networks, Vol.2, No 4, 197-221, Sept..1995

[7] P.Koorevaar, J.Ruprecht „Frequency Overlay of GSM and Cellular B-CDMA" Proc. IEEE VTC., 497-501, Stocholm, June 1996

[8] L.B.Milstein, D.L.Schilling „The CDMA Overlay concept” Proc. IEEE VTC, 476-480, Stockholm, June 1996

[9] P.Kaczorek, D.Rutkowski „A Comparison of Narrowband and Broadband Overlay of Cellular CDMA on GSM", paper accepted for presentation on IEEE VTC2000-Spring, Tokyo 2000

[10] P.Kaczorek, D.Rutkowski „A picocell CDMA Overlay on GSM system for Fast Data Transmission" paper accepted for presentation on Nat. Radio Comm. Conf. KKRR'2000, Poznań, Poland, June 2000

[11] A.Mehrotra „Cellular Radio Performance Engineering” Artech House, 1994 